Article

\title{
Dose-Limiting Organs at Risk in Carbon Ion Re-Irradiation of Head and Neck Malignancies: An Individual Risk-Benefit Tradeoff
}

\author{
Thomas Held ${ }^{1,2,3}$ (), Semi B. Harrabi 1,2,3,4 , Kristin Lang 1,2,3 , Sati Akbaba 1,2,3®D, \\ Paul Windisch 5 , Denise Bernhardt 1,2,3, Stefan Rieken 1,2,3,4,6,7, Klaus Herfarth 1,2,3,4,6,7,

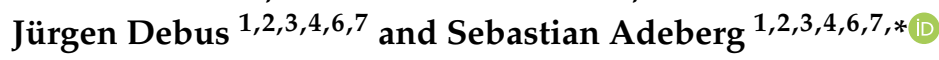 \\ 1 Heidelberg University Hospital, Department of Radiation Oncology, 69120 Heidelberg, Germany; \\ thomas.held@med.uni-heidelberg.de (T.H.); semi.harrabi@med.uni-heidelberg.de (S.B.H.); \\ kristin.lang@med.uni-heidelbeg.de (K.L.); sati.akbaba@med.uni-heidelberg.de (S.A.); \\ denise.bernhardt@med.uni-heidelberg.de (D.B.); stefan.rieken@med.uni-heidelberg.de (S.R.); \\ klaus.herfarth@med.uni-heidelberg.de (K.H.); juergen.debus@med.uni-heidelbeg.de (J.D.) \\ 2 Heidelberg Institute of Radiation Oncology (HIRO), 69120 Heidelberg, Germany \\ 3 National Center for Tumor diseases (NCT), 69120 Heidelberg, Germany \\ 4 Heidelberg Ion-Beam Therapy Center (HIT), 69120 Heidelberg, Germany \\ 5 University Hospital of Zurich, Department of Radiation Oncology, 8091 Zurich, Switzerland; \\ paul.windisch@med.uni-heidelberg.de \\ 6 Clinical Cooperation Unit Radiation Oncology, German Cancer Research Center (DKFZ), \\ 69120 Heidelberg, Germany \\ 7 German Cancer Consortium (DKTK), partner site Heidelberg, German Cancer Research Center (DKFZ), \\ 69120 Heidelberg, Germany \\ * Correspondence: sebastian.adeberg@med.uni-heidelberg.de
}

Received: 18 October 2019; Accepted: 5 December 2019; Published: 13 December 2019

check for updates

\begin{abstract}
Background: Carbon ion re-irradiation (CIR) was evaluated to investigate treatment planning and the consequences of individual risk-benefit evaluations concerning dose-limiting organs at risk (OAR). Methods: A total of 115 consecutive patients with recurrent head and neck cancer (HNC) were analyzed after initial radiotherapy and CIR at the same anatomical site. Toxicities were evaluated in line with the Common Terminology Criteria for Adverse Events 4.03. Results: The median maximum cumulative equivalent doses applied in fractions of 2 Gy (EQD2) to the brainstem, optic chiasm, ipsilateral optic nerve, and spinal cord were 56.8 Gy (range 0.94-103.9), 51.4 Gy (range 0-120.3 Gy), 63.6 Gy (range 0-146.1 Gy), and 28.8 Gy (range 0.2-87.7 Gy). The median follow up after CIR was 24.0 months (range $2.5-72.0$ months). The cumulative rates of acute and late severe ( $\geq$ grade III) side effects after CIR were $1.8 \%$ and $14.3 \%$. Conclusion: In recurrent HNC, an individual risk-benefit tradeoff is frequently inevitable due to unfavorable location of tumors in close proximity to vital OAR. There are uncertainties about the dose tolerance of OAR after CIR, which warrant increased awareness about the potential treatment toxicity and further studies on heavy ion re-irradiation.
\end{abstract}

Keywords: head and neck cancer; local recurrence; planning target volume; heavy ion therapy; dose constraints

\section{Introduction}

Re-irradiation in cases of head and neck cancer (HNC) is associated with an increased risk of severe side effects [1-3]. The total cumulative applied radiation dose, the irradiated volume [4], and the time interval [5-7] are crucial when considering a second course of irradiation at the same anatomical site. Nonetheless, toxicity rates are frequently underestimated, even in prospective clinical studies [8]. 
Other factors that influence the dose tolerance of organs at risk (OAR) are fractionation effects [9] and the simultaneous administration of systemic therapies [10]. In addition, location effects impact the probabilities of normal tissue complications since radiosensitive structures are not homogeneously distributed within organs [11]. Patient related co-factors such as smoking or age may increase the rate of side effects. Many uncertainties remain in regard to the effects of dose volume on the probabilities of complications of OAR after re-irradiation [12-14].

The high linear energy transfer of carbon ion radiotherapy results in greater relative biological effectiveness (RBE) than photons, which depends on the irradiated tissue [15]. Re-irradiation with heavy ions enables a favorable depth-dose distribution, which allows for escalation of the dose in a recurrent tumor while avoiding normal surrounding tissue and OAR [16,17]. Limiting the total dose applied to OAR is essential for preventing severe side effects, such as vision loss.

Data on carbon ion re-irradiation (CIR) are scarce, and there are currently no guidelines for dose constraints regarding OAR for the treatment of recurrent HNC with carbon ions. Uncertainties regarding the dose tolerance of normal tissue after heavy ion re-irradiation necessitate greater awareness of treatment-specific toxicities. Therefore, CIR was analyzed to investigate treatment planning and the effects of individual risk-benefit evaluations concerning OAR.

\section{Results}

\subsection{Patient Characteristics}

The most common tumor site was the paranasal sinuses ( $n=33,28.7 \%)$, followed by the salivary glands $(n=19,16.5 \%)$ and the nasopharynx $(n=22,19.1 \%)$. The vast majority of patients had adenoid cystic carcinomas (ACC, $n=77,67.0 \%$ ) or squamous cell carcinomas (SCC, $n=23,20.0 \%$ ). Prior to CIR, staging was conducted according to the 8th edition of the Union for International Cancer Control (UICC) tumor node metastasis (TNM) system. Tumor infiltration of the base of the skull or the immediate surroundings occurred in 94 patients $(n=94,81.7 \%)$. Detailed patient and tumor characteristics are shown in Table 1.

Table 1. Patient characteristics ( $n=115$ patients).

\begin{tabular}{ccc}
\hline Characteristics & Patients & \% \\
\hline Female/Male & $53 / 62$ & $46.1 / 53.9$ \\
ECOG status & & \\
0 & 61 & 53.0 \\
1 & 51 & 44.4 \\
2 & 3 & 2.6 \\
Histology & & \\
ACC & 77 & 67.0 \\
HNSCC & 23 & 20.0 \\
Adenocarcinoma & 9 & 7.8 \\
Other & 6 & 5.2 \\
Tumor Site & & \\
Paranasal sinuses & 33 & 28.7 \\
Nasopharynx & 22 & 19.1 \\
Salivary glands & 19 & 16.5 \\
Skull base & 13 & 11.3 \\
Oral cavity & 10 & 8.7 \\
Oropharynx & 8 & 7.0 \\
Other & 10 & 8.7 \\
TNM stage prior to CIR & & \\
T1-2 & 7 & 6.1 \\
T3-4 & 89 & 77.4 \\
\hline
\end{tabular}


Table 1. Cont

\begin{tabular}{ccc}
\hline Characteristics & Patients & $\mathbf{\%}$ \\
\hline TX & 19 & 16.5 \\
N0 & 81 & 70.4 \\
N1 & 3 & 2.6 \\
N2 & 13 & 11.3 \\
NX & 18 & 15.7 \\
M0 & 89 & 77.4 \\
M1 & 26 & 22.6
\end{tabular}

Abbreviations: Eastern Cooperative Oncology Group (ECOG), adenoid cystic carcinoma (ACC), head and neck squamous cell carcinoma (HNSCC), tumor node metastasis (TNM), carbon ion re-irradiation (CIR).

\subsection{Radiation Therapy}

\subsubsection{Initial Radiotherapy}

The first radiation treatment consisted of 3D-conformal RT in 8 patients (7.0\%), intensity-modulated radiation therapy (IMRT) in 39 patients $(33.9 \%)$, and bimodal RT (IMRT and carbon ion boost) in 60 patients $(52.2 \%)$. A total of six patients $(n=6,5.2 \%)$ received carbon ion radiotherapy as initial treatment. The irradiation technique was unspecified in two patients $(1.7 \%)$. Patients with bimodal RT received a median dose of 50 Gy (range 48-56 Gy) with photon IMRT and a median dose of 24 Gy (RBE) (range 18-24 Gy (RBE)) with carbon ions. A total of seven patients $(n=7,6.1 \%)$ received two prior irradiations at the same anatomical site.

\subsubsection{Carbon Ion Re-Irradiation}

The median interval between the previous course of irradiation and CIR was 3.4 years (range 0.3-13.3 years). A median total dose of 51 Gy (RBE) (range 39-60 Gy (RBE)) in fractions of 3 Gy (RBE) was applied. The median PTV and CTV were $128.9 \mathrm{cc}$ (range 13.3-925.0 cc) and $83.1 \mathrm{cc}$ (range 6.3-710.5 cc), respectively. No simultaneous systemic therapy was applied during CIR. The equivalent doses in 2-Gy fractions (EQD2) were calculated with an assumed $\alpha / \beta$ of 2 for ACC and 10 for SCC and other tumor entities according to the local effect model (LEM I). The median cumulative EQD2 after initial irradiation and CIR was 135.8 Gy (range 101.3-155.5 Gy). Detailed information about the initial RT and CIR are shown in Table 2.

Table 2. Treatment characteristics ( $n=115$ patients).

\begin{tabular}{ccc}
\hline Characteristics & Patients & $\mathbf{\%}$ \\
\hline Initial RT & & \\
Bimodal RT & 60 & 52.2 \\
IMRT & 39 & 33.9 \\
3DCRT & 8 & 7.0 \\
CIRT & 6 & 5.2 \\
Unspecified & 2 & 1.7 \\
Yes & 10 & \\
No & 105 & 91.7 \\
& Median & Range \\
Carbon ion re-irradiation & & \\
Total dose [Gy (RBE)] & 51.0 & $39.0-60.0$ \\
Total dose [EQD2] & 63.8 & $48.8-75.0$ \\
Fractions & 17 & $13-20$ \\
Planning target volume & 128.9 & $13.3-925.0$ \\
Clinical target volume & 83.1 & $6.3-710.5$ \\
\hline
\end{tabular}


Table 2. Cont.

\begin{tabular}{ccc}
\hline Characteristics & Patients & $\mathbf{\%}$ \\
\hline Further treatment parameters & & \\
Cumulative Dose [EQD2] & 135.8 & $101.3-155.0$ \\
RT-Interval [years] & 3.4 & $0.3-13.3$ \\
Prior tumor-specific treatments & 2 & $1-8$ \\
\hline
\end{tabular}

Abbreviations: Radiotherapy (RT), intensity modulated radiation therapy (IMRT), IMRT and carbon ion boost (bimodal RT), 3D conformal radiation therapy (3DCRT), carbon ion radiotherapy (CIRT), equivalent dose in $2 \mathrm{~Gy}$ fractions (EQD2), relative biological effectiveness (RBE).

\subsubsection{Organs at Risk}

For the optic nerves, temporal lobes, and mandibular joints, only the total dose to the ipsilateral side was considered for lateralized tumors. The median maximum cumulative EQD2 applied to the brainstem, optic chiasm, ipsilateral optic nerve, and spinal cord was 56.8 Gy (range 0.94-103.9), 51.4 Gy (range 0-120.3 Gy), 63.6 Gy (range 0-146.1 Gy), and 28.8 Gy (range 0.2-87.7 Gy), respectively. Information about the irradiated volume of the respective OAR was not specified.

Generally, the maximum applied dose was limited to the surface area. However, in 49 patients ( $n=49,42.6 \%$ ), the orbital cavity was infiltrated by the tumor. Consequently, the maximum dose constraint for the ipsilateral optic nerve was disregarded in individual cases upon patient consent to achieve improved tumor control. In these patients, the median maximum cumulative EQD2 applied to the ipsilateral optic nerve and optic chiasm was 113.1 Gy (range 65.5-146.1 Gy) and 66.8 Gy (range 14.5-120.3 Gy), respectively.

When these cases were excluded ( $n=66$ patients, $57.4 \%)$, the maximum cumulative EQD2 applied to the ipsilateral optic nerve and optic chiasm was 42.4 Gy (range 0-102.7 Gy) and 27.4 Gy (range 0-93.6 Gy), respectively. The total dose of CIR applied to the brainstem, optic nerve, and spinal cord was $20 \%$ higher if the RT interval was more than two years. Further information on the total dose applied to OAR is shown in Table 3.

Table 3. Organs at risk $(n=115$ patients).

\begin{tabular}{|c|c|c|c|c|c|c|c|c|}
\hline \multirow[t]{2}{*}{ OAR } & \multicolumn{2}{|c|}{ Initial RT [EQD2] } & \multicolumn{2}{|c|}{ CIR [Gy (RBE)] } & \multicolumn{2}{|c|}{ CIR [EQD2] } & \multicolumn{2}{|c|}{$\begin{array}{c}\text { Initial RT+CIR } \\
\text { [EQD2] }\end{array}$} \\
\hline & Median & Range & Median & Range & Median & Range & Median & Range \\
\hline \multicolumn{9}{|l|}{ Mean } \\
\hline Brainstem & 16.6 & $0-39.8$ & 1.5 & $0-16.6$ & 0.8 & $0-12.1$ & 17.7 & $0-46.3$ \\
\hline Optic chiasm & 14.3 & $0-53.8$ & 3.2 & $0-46.0$ & 2.0 & $0-52.5$ & 18.4 & $0-87.3$ \\
\hline Optic nerve & 26.3 & $0-74.0$ & 12.8 & $0-57.1$ & 9.5 & $0-68.6$ & 38.4 & $0-124.0$ \\
\hline Optic nerve * & 8.4 & $0-66.7$ & 0.7 & $0-38.2$ & 0.4 & $0-40.1$ & 15.2 & $0-68.1$ \\
\hline Brain & n.a. & n.a. & 9.8 & $0-29.4$ & 6.3 & $0-27.4$ & n.a. & n.a. \\
\hline Mandibular joint & n.a. & n.a. & 27.0 & $0-59.6$ & 25.2 & $0-67.0$ & n.a. & n.a. \\
\hline Spinal cord & 9.3 & $0-48.0$ & 0.1 & $0-4.2$ & 0.1 & $0-2.3$ & 9.3 & $0-49.5$ \\
\hline \multicolumn{9}{|l|}{ Maximum } \\
\hline Brainstem & 45.6 & $0-67.8$ & 19.7 & $0-49.8$ & 15.6 & $0-59.3$ & 56.8 & 0.9-103.9 \\
\hline Optic chiasm & 30.7 & $0-60.2$ & 17.3 & $0-56.1$ & 14.1 & $0-70.7$ & 51.4 & $0-120.3$ \\
\hline Optic nerve & 46.2 & $0-76.5$ & 28.5 & $0-64.7$ & 26.4 & $0-83.0$ & 63.3 & $0-146.1$ \\
\hline Optic nerve * & 21.4 & $0-71.0$ & 6.6 & $0-50.9$ & 4.4 & $0-61.1$ & 42.4 & $0-102.7$ \\
\hline Brain & n.a. & n.a. & 52.8 & $0-64.0$ & 67.6 & 0-88.9 & n.a. & n.a. \\
\hline Mandibular joint & n.a. & n.a. & 43.1 & $0-59.6$ & 50.2 & $0-72.0$ & n.a. & n.a. \\
\hline Spinal cord & 27.8 & $0-52.3$ & 1.2 & $0-53.8$ & 0.6 & 0-69.6 & 28.8 & $0.2-87.7$ \\
\hline
\end{tabular}

Abbreviations: organ at risk (OAR), Radiotherapy (RT), carbon ion re-irradiation (CIR), equivalent dose in 2 Gy fractions (EQD2), * excluding 49 patients with tumors infiltrating the orbital cavity.

\subsection{Acute and Late Toxicity}

The median follow up after CIR was 24.0 months (range 2.5-72.0 months). Acute toxicities included new or worsening adverse events that were attributable to CIR and occurred from the start 
until 90 days after radiation treatment. All patients completed re-irradiation without interruptions. The cumulative rate of acute severe toxicity ( $\geq$ grade III) was $1.8 \%$. There were no cases of grade IV or $\mathrm{V}$ acute toxicity. Low grades of acute toxicity (grades I-II) most commonly included radiation dermatitis, oral mucositis, and xerostomia. Several patients developed hearing impairment due to unilateral middle-ear inflammation. Furthermore, neurological side effects such as trigeminal nerve disorder were common.

Late toxicity was observed in 70 patients $(60.9 \%)$ with available long-term clinical follow up and imaging. The cumulative rate of late severe toxicity was $14.3 \%$. High-grade ( $\geq$ grade III) optic nerve disorder occurred in four patients. In two of these patients, the orbital cavity was infiltrated by the tumor. After obtaining patient consent, the maximum dose constraint for the ipsilateral optic nerve was disregarded to increase the tumor control rate. The time intervals from the start of CIR until the occurrence of high-grade optic nerve disorder in these patients were 2.4 and 16.3 months for grade III and 7.7 and 40.1 months for grade IV, respectively.

In six patients $(5.2 \%)$, an enucleation of the eye was performed prior to CIR due to tumor infiltration. Grade II and III necrosis of the central nervous system (CNS) occurred in $9(7.8 \%)$ and 4 $(3.5 \%)$ patients, respectively. The management of CNS necrosis after CIR is beyond the scope of this study [3].

Dose limits for the optic nerves and temporal lobes were surpassed in all of the patients with severe late ( $\geq$ grade III) optic nerve disorder $(n=4)$ and CNS necrosis $(n=4)$. In addition, all patients with a maximum cumulative dose beyond the constraints [18] of 54 Gy for the optic system $(n=39)$, $60 \mathrm{~Gy}$ for the temporal lobe $(n=22)$, and long-term clinical follow up and imaging were analyzed. Of these patients, $10.3 \%(n=4)$ and $18.2 \%(n=4)$ developed optic nerve disorder and CNS necrosis, respectively. Data on acute and late toxicity are shown in Table 4.

Table 4. Acute and late treatment related toxicity.

\begin{tabular}{|c|c|c|}
\hline Toxicity & $n=$ & $\%$ \\
\hline \multicolumn{3}{|l|}{$\begin{array}{c}\text { Acute toxicity } \\
\text { High grade (CTCAE III-IV) }\end{array}$} \\
\hline Dysphagia ${ }^{\circ} \mathrm{III}$ & 1 & 0.9 \\
\hline $\begin{array}{l}\text { Hearing impairment }{ }^{\circ} \mathrm{III} \\
\text { Low grade }(\text { CTCAE I-II) }\end{array}$ & 1 & 0.9 \\
\hline Dermatitis radiation ${ }^{\circ} \mathrm{I}-\mathrm{II}$ & 38 & 33.1 \\
\hline Mucositis oral ${ }^{\circ} \mathrm{I}-\mathrm{II}$ & 30 & 26.1 \\
\hline Xerostomia ${ }^{\circ} \mathrm{I}-\mathrm{II}$ & 23 & 20.0 \\
\hline Headache ${ }^{\circ} \mathrm{I}-\mathrm{II}$ & 14 & 12.1 \\
\hline Alopecia ${ }^{\circ} \mathrm{I}$ & 11 & 9.6 \\
\hline Hearing impairment ${ }^{\circ} \mathrm{I}-\mathrm{II}$ & 11 & 9.5 \\
\hline Dysgeusia ${ }^{\circ} \mathrm{I}-\mathrm{II}$ & 10 & 8.7 \\
\hline Trismus ${ }^{\circ} \mathrm{I}$ & 10 & 8.7 \\
\hline Dysphagia ${ }^{\circ} \mathrm{I}-\mathrm{II}$ & 9 & 7.8 \\
\hline Middle ear inflammation ${ }^{\circ} \mathrm{I}-\mathrm{II}$ & 9 & 7.8 \\
\hline Conjunctivitis ${ }^{\circ} \mathrm{I}-\mathrm{II}$ & 6 & 5.2 \\
\hline Weight loss ${ }^{\circ} \mathrm{I}-\mathrm{II}$ & 4 & 3.5 \\
\hline Trigeminal nerve disorder ${ }^{\circ} \mathrm{I}-\mathrm{II}$ & 3 & 2.8 \\
\hline Optic nerve disorder ${ }^{\circ} \mathrm{I}-\mathrm{II}$ & 3 & 2.8 \\
\hline \multicolumn{3}{|l|}{ Late toxicity } \\
\hline Optic nerve disorder ${ }^{\circ} \mathrm{III}-\mathrm{IV}$ & 4 & 5.7 \\
\hline Hearing impairment ${ }^{\circ} \mathrm{III}$ & 2 & 2.9 \\
\hline CNS necrosis ${ }^{\circ} \mathrm{III}$ & 4 & 5.7 \\
\hline \multicolumn{3}{|l|}{ Low grade (CTCAE I-II) } \\
\hline CNS necrosis ${ }^{\circ} \mathrm{I}-\mathrm{II}$ & 21 & 30 \\
\hline Xerostomia ${ }^{\circ} \mathrm{I}-\mathrm{II}$ & 14 & 20.0 \\
\hline
\end{tabular}


Table 4. Cont.

\begin{tabular}{ccc}
\hline Toxicity & $n=$ & $\%$ \\
\hline Hearing impairment $^{\circ} \mathrm{I}-\mathrm{II}$ & 12 & 17.1 \\
Trismus $^{\circ} \mathrm{I}$ & 10 & 14.3 \\
Dysgeusia $^{\circ} \mathrm{I}-\mathrm{II}$ & 10 & 14.5 \\
Middle ear inflammation $^{\circ} \mathrm{I}-\mathrm{II}$ & 9 & 12.9 \\
Headache $^{\circ} \mathrm{I}-\mathrm{II}$ & 7 & 10.0 \\
Trigeminal nerve disorder $^{\circ} \mathrm{I}-\mathrm{II}$ & 7 & 10.0 \\
Dysphagia $^{\circ} \mathrm{I}-\mathrm{II}$ & 5 & 7.2 \\
Alopecia $^{\circ} \mathrm{I}$ & 5 & 7.1 \\
Weight loss $^{\circ} \mathrm{I}-\mathrm{II}$ & 3 & 4.3 \\
Osteonecrosis $^{\circ} \mathrm{I}-\mathrm{II}$ & 3 & 4.3 \\
Optic nerve disorder $^{\circ} \mathrm{I}-\mathrm{II}$ & 2 & 2.9 \\
\hline
\end{tabular}

Abbreviations: Common Terminology Criteria for Adverse Events (CTCAE), central nervous system (CNS).

\section{Discussion}

Patients with recurrent HNC frequently received a total cumulative dose that was above the maximum dose constraints for OAR such as the temporal lobe, depending on the radiotherapy interval. In these cases, a risk-benefit trade off was inevitable due to an unfavorable tumor location near OAR. Nonetheless, the cumulative rate of acute and late severe toxicity of $14.3 \%$ was acceptable. In most patients with high-grade ( $\geq$ grade III) late toxicity, the dose limits were surpassed.

In patients with tumors infiltrating the orbital cavity $(n=49,42.6 \%)$, dose constraints for the ipsilateral optic nerve were disregarded upon patient consent to achieve improved tumor control. Of these patients, $10.3 \%(n=4)$ developed optic nerve disorder ( $\geq$ grade III). Considering the relatively short median follow up of 24.0 months, the expected rate of late adverse events will probably increase over time in these patients. Contrary to the assumptions of treatment planning, the exact dose maxima of the initial RT and CIR were not entirely congruent in the current analysis. Therefore, dose-volume effects were probably equally critical for preventing treatment toxicity. In patients without orbital cavity infiltration, the median maximum cumulative EQD2 applied to the ipsilateral optic nerve (42.4 Gy) was within dose limits.

The RT interval is another substantial factor that impacts the risk-benefit evaluation prior to CIR $[19,20]$. The total dose of CIR applied to OAR was $20 \%$ higher if the minimum RT interval was more than two years. Considering all patients for whom dose constraints were exceeded intentionally ( $n=49,42.6 \%)$, the dose prescription at CIR was restrictive in the current analysis. Hayashi et al. [21] reported absolute dose constraints at CIR of $30 \mathrm{~Gy}$ (RBE) for the brainstem and spinal cord and $40 \mathrm{~Gy}$ (RBE) for the optic system. Hu et al. [6] assumed a recovery from the previous photon radiotherapy set at 70\%, independent of the RT interval. Consequently, the CTV was reduced if it was close to OAR. However, the irradiated volume of the respective OAR was not specified in the current evaluation.

Currently, there are no guidelines regarding the dose tolerance of OAR after heavy ion re-irradiation. Therefore, a thorough risk-benefit evaluation is required for each individual patient. The potential for severe early and late side effects after CIR depends primarily on the location of the tumor. In particular, re-irradiation of tumors that have infiltrated the base of the skull result in higher doses applied to dose-limiting OAR, such as the brainstem and temporal lobes [22]. Furthermore, simultaneous systemic therapies lead to a significant increase in treatment-related toxicities [1,2]. However, in prior evaluations on heavy-ion re-irradiation $[3,6,23]$, concomitant systemic therapies were primarily excluded. The clinical effects of CIR in combination with systemic agents have yet to be evaluated.

According to this current data analysis and guided by the clinical experience in CIR of recurrent HNC at our institution since 2010, several constraints of dose-limiting OAR can be pointed out. If the RT interval is less or equal to two years, the proposed cumulative radiation tolerance of the optic system and brain stem complies to the QUANTEC [13] analyses. However, if the RT interval is more than two years, an increase of the total cumulative dose to OAR by up to $20 \%$ has shown acceptable 
toxicity profiles [20]. Based on these results, proposed dose limits for CIR are provided in Table 5. Individual dose prescription can differ and will be at the discretion of the radiation oncologist.

Table 5. Proposed radiation tolerance of organs at risk for carbon ion re-irradiation.

\begin{tabular}{|c|c|c|c|}
\hline OAR & $\begin{array}{c}\text { Maximum Cumulative } \\
\text { BED2Gy(RT interval } \leq \\
2 \text { years })\end{array}$ & $\begin{array}{c}\text { Maximum Cumulative } \\
\text { BED2Gy(RT interval > } \\
2 \text { years })\end{array}$ & Comment \\
\hline Brain stem $(\alpha / \beta=2)$ & 60 & $72(\hat{=}+20 \%)$ & Maximum (surface) \\
\hline Optic chiasm $(\alpha / \beta=3)$ & 54 & $64.8(\hat{=}+20 \%)$ & Maximum \\
\hline Optic nerves $(\alpha / \beta=3)$ & 54 & $64.8(\hat{=}+20 \%)$ & Maximum \\
\hline Spinal cord $(\alpha / \beta=2)$ & 50 & $60(\hat{=}+20 \%)$ & Maximum \\
\hline $\begin{array}{l}\text { Jawbone, inner ear, skin, } \\
\text { mandibular joint, } \\
\text { mandibular bone, lacrimal } \\
\text { gland, parotid gland, } \\
\text { submandibular gland, eyes, } \\
\text { internal carotid arteries, } \\
\text { temporal lobes }\end{array}$ & \multicolumn{2}{|c|}{ ALARA * } & 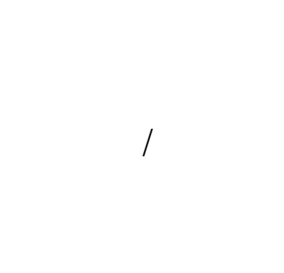 \\
\hline
\end{tabular}

Abbreviations: organ at risk (OAR), biological effective dose in 2 Gy fractions (BED2Gy), radiotherapy (RT), as low as reasonably achievable (ALARA).

The time intervals until the occurrence of high-grade toxicities after CIR varied considerably. The onset of optic nerve disorders determined from the start of CIR ranged from 2.4-40.1 months. Possible explanations for this finding include the impact of volume and location effects, as well as the total dose applied in the initial radiation treatment. In contrast, the onset of CNS necrosis after CIR followed a less elusive pattern. Most tumors infiltrated the base of the skull, so the median maximum dose of 52.8 Gy (RBE) (range 0-64.0 Gy (RBE)) applied to the temporal lobe at CIR was extensive. Grades I, II, and III CNS necrosis occurred after median time intervals of 8.8 (range 5.5-75.0 months), 8.2 (range 2.3-25.5 months), and 14.2 months (range 8.7-32.5 months), respectively. The clinical outcomes with early and adequate treatment were encouraging [3].

The cumulative rates of acute and late severe ( $\geq$ grade III) side effects after CIR were acceptable at $1.8 \%$ and $14.3 \%$, respectively. Prior studies on re-irradiation of HNC with carbon ions reported similar toxicity profiles $[6,23,24]$. However, the distribution of re-irradiation sites in our assessment was quite different from those of prior studies on CIR in recurrent HNC. In this study, 49 patients $(42.6 \%)$ presented with tumor infiltration of the orbital cavity, and 94 patients $(81.7 \%)$ had tumors with infiltration of the base of the skull or the immediate surroundings. Furthermore, compared to prior studies on re-irradiation with heavy ions [21], the median PTV of $128.9 \mathrm{cc}$ in this evaluation was extensive, reaching about $50-100 \%$ more. In addition, the tumor locations were possibly more challenging in the current analysis ( $81.7 \%$ of them infiltrated the base of the skull).

This analysis had several limitations worth mentioning. First, the rates of treatment toxicities could have been underestimated due to the retrospective design of the study. In a prospective setting, side effects related to CIR might be reported more frequently. Particularly, long-term sequelae and chronic late radiation effects were underrated since several patients were lost to follow up due to the poor prognosis. Second, the heterogeneity of the initial radiation treatment limits the validity of our results. Third, only the total dose applied to OAR was recorded, and volume effects were not considered. In addition, differences remain in the fractionation and uncertainties of the RBE of carbon ions in the clinical setting.

Despite the limitations, the analysis was independently validated by several experts in the field of heavy ion therapy. Furthermore, a comparatively large patient cohort $(n=115)$ was analyzed in regard to dose-limiting OAR, considering the scarcity of data on CIR in recurrent head-and-neck malignancies. To evaluate CIR in recurrent HNC further, a treatment plan comparison including photon and proton plans is currently being prepared. 


\section{Materials and Methods}

\subsection{Patient Characteristics}

After approval by the local ethics committee (S-421/2015), patients from the cancer database of the National Center for Tumor Diseases (NCT) with head and neck cancer (HNC) were screened. All patients with at least one prior course of radiotherapy (RT) and CIR at the same anatomical site were evaluated. Patients without complete information on the total cumulative dose applied to the optic system, brainstem, and spinal cord were excluded from this retrospective study. A total of 115 ( $n=$ 115) patients with HNC treated at our clinic with CIR between 2010 and 2018 were analyzed, and 10 patients ( $n=10,8.7 \%$ ) received surgical resection prior to CIR.

\subsection{Treatment Planning}

Patients were immobilized with a thermoplastic head mask. Computed tomography (CT) scans (3-mm slice thickness) were used for treatment planning, and contrast-enhanced T1-weighted magnetic resonance imaging (MRI) was used for image registration. Treatment planning was conducted using Syngo PT Planning version 13 (Siemens ${ }^{\circledR}$, Erlangen, Germany). The clinical target volume (CTV) included the gross tumor volume in contrast-enhanced CT or MRI with an additional margin of 2-5 $\mathrm{mm}$. In patients with surgical resection prior to CIR, the resection cavity was included in the CTV. No elective lymph node treatment was performed. A safety margin of 2-3 mm was added for the planning target volume (PTV), depending on the patient positioning and beam arrangement.

Prior to CIR, the cumulative dose to be applied was determined for OAR (the optic system, brainstem, and spinal cord). To account for differences in fractionation, an assumed $\alpha / \beta$ of 2 was used for the brainstem, temporal lobe, and spinal cord. For the optic system and mandibular joint, $\alpha / \beta$ values of 3 and 3.5 were used for dose calculation. In individual cases, the CTV was adapted by the treating radiation oncologist to reduce the cumulative dose applied to vital OAR. Treatment was performed at our clinic using the active raster scanning method with daily image guidance by orthogonal X-rays, as well as routine CT scans and position correction in six degrees of freedom.

\subsection{Follow Up}

Imaging included contrast-enhanced MRI or CT scans of the head and neck, which were scheduled six to eight weeks after CIR and then every three months thereafter within the first year after treatment. Symptoms and toxicities related to treatment were recorded in a non-standardized manner by a clinician at each follow-up visit. Patients also presented regularly to an ear, nose, and throat specialist or oral and maxillofacial specialist for clinical examination.

\subsection{Statistics}

Statistical analyses were conducted using SPSS Statistics 25 (IBM ${ }^{\circledR}$, New York, USA) and the software R version 3.4.3 (www.r-project.org). The median follow-up was calculated using the inverse Kaplan-Meier method. Toxicities were evaluated from the patients' medical records in line with the Common Terminology Criteria for Adverse Events (CTCAE) 4.03.

\section{Conclusions}

In recurrent $\mathrm{HNC}$, a risk-benefit tradeoff is frequently inevitable due to an unfavorable location of the tumor near OAR. Dose constraints may be exceeded in individual dose prescriptions in CIR to improve local tumor control after obtaining patient consent. Since there are uncertainties about the dose tolerance of OAR after CIR, increased awareness for potential treatment toxicity and further studies on heavy ion re-irradiation are necessary.

Author Contributions: T.H.: Data curation, statistical analysis, investigation, validation, methodology, visualization, writing original draft, writing review, editing. K.L., S.A. (Sati Akbaba), P.W.: Data curation, 
investigation, validation, methodology, editing. S.B.H., D.B., S.R., K.H., J.D.: Data curation, validation, methodology, project administration, supervision, editing. S.A. (Sebastian Adeberg): Data curation, statistical analysis, investigation, validation, methodology, visualization, writing original draft, writing review, project administration, supervision, editing.

Funding: This research received no external funding.

Conflicts of Interest: Denise Bernhardt reports grants and personal fees from Roche, Novocure, and Accuray Inc. as well as grants and personal fees from Medizinische Fakultät Heidelberg, Arbeitsgemeinschaft Radioonkologie, and Deutsche Krebshilfe, outside the submitted work. Jürgen Debus reports grants from The Clinical Research Institue GmbH, View Ray Inc., Accuray International SARL, Accuray Inc., RaySearch Laboratories AB, Vision RT limited, Merck Serono GmbH, Astellas Pharma GmbH, Astra Zeneca GmbH, Siemens Healthcare GmbH, Merck KGaA Accounts Payable, Solution Akademie GmbH, Ergomed PLC Surrey Research Park, Siemens Healthcare $\mathrm{GmbH}$, Quintiles GmbH, Pharmaceutecal Research Associates GmbH, Boehringer Ingelheim Pharma GmbH \& Co. KG, PTW-Freiburg Dr. Pychlau GmbH, Nanobiotix A.A., outside the submitted work. Sebastian Adeberg reports personal fees from Merck Serono $\mathrm{GmbH}$ and Accuray Inc., outside the submitted work. The other authors declare no conflict of interest.

\section{References}

1. Spencer, S.A.; Harris, J.; Wheeler, R.H.; Machtay, M.; Schultz, C.; Spanos, W.; Rotman, M.; Meredith, R.; Ang, K.K. Final report of RTOG 9610, a multi-institutional trial of reirradiation and chemotherapy for unresectable recurrent squamous cell carcinoma of the head and neck. Head Neck 2008, 30, 281-288. [CrossRef] [PubMed]

2. Langer, C.J.; Harris, J.; Horwitz, E.M.; Nicolaou, N.; Kies, M.; Curran, W.; Wong, S.; Ang, K. Phase II study of low-dose paclitaxel and cisplatin in combination with split-course concomitant twice-daily reirradiation in recurrent squamous cell carcinoma of the head and neck: Results of Radiation Therapy Oncology Group Protocol 9911. J. Clin. Oncol. 2007, 25, 4800-4805. [CrossRef] [PubMed]

3. Held, T.; Akbaba, S.; Lang, K.; Harrabi, S.; Bernhardt, D.; Freudlsperger, C.; Kargus, S.; Plinkert, P.; Rieken, S.; Herfarth, K.; et al. Clinical Management of Blood(-)Brain Barrier Disruptions after Active Raster-Scanned Carbon Ion Re-Radiotherapy in Patients with Recurrent Head-and-Neck Cancer. Cancers 2019, 11, 383. [CrossRef] [PubMed]

4. Emami, B.; Lyman, J.; Brown, A.; Coia, L.; Goitein, M.; Munzenrider, J.E.; Shank, B.; Solin, L.J.; Wesson, M. Tolerance of normal tissue to therapeutic irradiation. Int. J. Radiat. Oncol. Boil. Phys. 1991, 21, 109-122. [CrossRef]

5. Ang, K.K.; Price, R.E.; Stephens, L.C.; Jiang, G.L.; Feng, Y.; Schultheiss, T.E.; Peters,, L.J. The tolerance of primate spinal cord to re-irradiation. Int. J. Radiat. Oncol. Boil. Phys. 1993, 25, 459-464. [CrossRef]

6. Hu, J.; Bao, C.; Gao, J.; Guan, X.; Hu, W.; Yang, J.; Hu, C.; Kong, L.; Lu, J.J. Salvage treatment using carbon ion radiation in patients with locoregionally recurrent nasopharyngeal carcinoma: Initial results. Cancer 2018, 124, 2427-2437. [CrossRef]

7. Kim, Y.S. Reirradiation of head and neck cancer in the era of intensity-modulated radiotherapy: Patient selection, practical aspects, and current evidence. Radiat. Oncol. J. 2017, 35, 1-15. [CrossRef]

8. Trotti, A.; Bentzen, S.M. The Need for Adverse Effects Reporting Standards in Oncology Clinical Trials. J. Clin. Oncol. 2004, 22, 19-22. [CrossRef]

9. De Felice, F.; Polimeni, A.; Valentini, V.; Brugnoletti, O.; Cassoni, A.; Greco, A.; de Vincentiis, M.; Tombolini, V. Radiotherapy Controversies and Prospective in Head and Neck Cancer: A Literature-Based Critical Review. Neoplasia 2018, 20, 227-232. [CrossRef]

10. Bentzen, S.M.; Rosenthal, D.I.; Weymuller, E.A.; Trotti, A. Increasing toxicity in nonoperative head and neck cancer treatment: Investigations and interventions. Int. J. Radiat. Oncol. Boil. Phys. 2007, 69 (Suppl. 2), S79-S82. [CrossRef]

11. Hopewell, J.W.; Trott, K.R. Volume effects in radiobiology as applied to radiotherapy. Radiother. Oncol. J. Eur. Soc. Ther. Radiol Oncol. 2000, 56, 283-288. [CrossRef]

12. Van Luijk, P.; Bijl, H.P.; Konings, A.W.; van der Kogel, A.J.; Schippers, J.M. Data on dose-volume effects in the rat spinal cord do not support existing NTCP models. Int. J. Radiat. Oncol. Boil. Phys. 2005, 61, 892-900. [CrossRef] [PubMed] 
13. Bentzen, S.M.; Constine, L.S.; Deasy, J.O.; Eisbruch, A.; Jackson, A.; Marks, L.B.; Ten Haken, R.K.; Yorke, E.D. Quantitative Analyses of Normal Tissue Effects in the Clinic (QUANTEC): An introduction to the scientific issues. Int. J. Radiat. Oncol. Boil. Phys. 2010, 76 (Suppl. 3), S3-S9. [CrossRef] [PubMed]

14. Das, S.; Patro, K.; Mukherji, A. Recovery and tolerance of the organs at risk during re-irradiation. J. Curr. Oncol. 2018, 1, 23-28.

15. Karger, C.P.; Peschke, P. RBE and related modeling in carbon-ion therapy. Phys. Med. Biol. 2017, 63, 1-2. [CrossRef]

16. Kanai, T.; Endo, M.; Minohara, S.; Miyahara, N.; Koyama-ito, H.; Tomura, H.; Matsufuji, N.; Futami, Y.; Fukumura, A.; Hiraoka, T.; et al. Biophysical characteristics of HIMAC clinical irradiation system for heavy-ion radiation therapy. Int. J. Radiat. Oncol. Boil. Phys. 1999, 44, 201-210. [CrossRef]

17. Eekers, D.B.P.; Roelofs, E.; Jelen, U.; Kirk, M.; Granzier, M.; Ammazzalorso, F.; Ahn, P.H.; Janssens, G.; Hoebers, F.J.P.; Friedmann, T.; et al. Benefit of particle therapy in re-irradiation of head and neck patients. Results of a multicentric in silico ROCOCO trial. Radiother. Oncol. J. Eur. Soc. Ther. Radiol. Oncol. 2016, 121, 387-394. [CrossRef]

18. Lambrecht, M.; Eekers, D.B.P.; Alapetite, C.; Burnet, N.G.; Calugaru, V.; Coremans, I.E.M.; Fossati, P.; Hoyer, M.; Langendijk, J.A.; Mendez Romero, A.; et al. Radiation dose constraints for organs at risk in neuro-oncology; the European Particle Therapy Network consensus. Radiother. Oncol. J. Eur. Soc. Ther. Radiol. Oncol. 2018, 128, 26-36. [CrossRef]

19. Duprez, F.; Madani, I.; Bonte, K.; Boterberg, T.; Vakaet, L.; Derie, C.; De Gersem, W.; De Neve, W. Intensity-modulated radiotherapy for recurrent and second primary head and neck cancer in previously irradiated territory. Radiother. Oncol. J. Eur. Soc. Ther. Radiol. Oncol. 2009, 93, 563-569. [CrossRef]

20. Held, T.; Windisch, P.; Akbaba, S.; Lang, K.; El Shafie, R.; Bernhardt, D.; Plinkert, P.; Kargus, S.; Rieken, S.; Herfarth, K.; et al. Carbon Ion Reirradiation for Recurrent Head and Neck Cancer: A Single-Institutional Experience. Int. J. Radiat. Oncol. Boil. Phys. 2019, 105, 803-811. [CrossRef]

21. Hayashi, K.; Koto, M.; Ikawa, H.; Hagiwara, Y.; Tsuji, H.; Ogawa, K.; Kamada, T. Feasibility of Re-irradiation using carbon ions for recurrent head and neck malignancies after carbon-ion radiotherapy. Radiother. Oncol. J. Eur. Soc. Ther. Radiol. Oncol. 2019, 136, 148-153. [CrossRef] [PubMed]

22. El Shafie, R.A.; Czech, M.; Kessel, K.A.; Habermehl, D.; Weber, D.; Rieken, S.; Bougatf, N.; Jakel, O.; Debus, J.; Combs, S.E. Evaluation of particle radiotherapy for the re-irradiation of recurrent intracranial meningioma. Radiat. Oncol. 2018, 13, 86. [CrossRef] [PubMed]

23. Jensen, A.D.; Poulakis, M.; Nikoghosyan, A.V.; Chaudhri, N.; Uhl, M.; Munter, M.W.; Herfarth, K.K.; Debus, J. Re-irradiation of adenoid cystic carcinoma: Analysis and evaluation of outcome in 52 consecutive patients treated with raster-scanned carbon ion therapy. Radiother. Oncol. J. Eur. Soc. Ther. Radiol. Oncol. 2015, 114, 182-188. [CrossRef] [PubMed]

24. Gao, J.; Hu, J.; Guan, X.; Yang, J.; Hu, W.; Kong, L.; Lu, J.J. Salvage Carbon-Ion Radiation Therapy for Locoregionally Recurrent Head and Neck Malignancies. Sci. Rep. 2019, 9, 4259. [CrossRef] 